\title{
Estudo comparativo entre estibogluconato de sódio BP 88R e antimoniato de meglumina no tratamento da leishmaniose cutânea: I. Eficácia e segurança
}

\author{
Comparative study between sodium stibogluconate BP $88^{\mathrm{R}}$ and meglumine \\ antimoniate for cutaneous leishmaniasis treatment: I. Efficacy and safety
}

\author{
Ana Cristina R. Saldanha, Gustavo Adolfo Sierra Romero, Edgar Merchan-Hamann, \\ Albino Verçosa Magalhães e Vanize de Oliveira Macedo
}

\begin{abstract}
Resumo A eficácia e segurança do antimoniato de meglumina e do estibogluconato de sódio BP 88R foram comparadas no tratamento da leishmaniose cutânea em Corte de Pedra, Bahia, área endêmica de leishmaniose causada por Leishmania (Viannia) braziliensis. Realizou-se um estudo quase-experimental que incluiu 127 pacientes cujo diagnóstico baseou-se na observação clínica e a intradermorreação de Montenegro. Cinqüenta e oito pacientes receberam antimoniato de meglumina e 69 estibogluconato de sódio. Utilizou-se a dose de $20 \mathrm{mg} / \mathrm{Sbv} / \mathrm{kg} / \mathrm{dia}$ por 20 dias, em ambos os grupos. Os pacientes foram acompanhados a cada dez dias durante 0 tratamento e mensalmente por três meses. Observou-se a cura em $62 \%$ dos pacientes tratados com antimoniato de meglumina e em 55\% daqueles tratados com estibogluconato de sódio $(p=0,42)$. A cefaléia foi mais freqüente na primeira metade do tratamento no grupo tratado com estibogluconato de sódio $(p=0,026)$. Na segunda metade do tratamento, os pacientes tratados com estibogluconato de sódio apresentaram maior freqüencia de mialgia/artralgia ( $p$ $=0,004)$ e dor abdominal/anorexia $(p=0,004)$. Três pacientes tratados com o estibogluconato de sódio apresentaram efeitos colaterais graves.
\end{abstract}

Palavras-chaves: Leishmaniose cutânea. Antimoniato de meglumina. Estibogluconato de sódio. Antimoniais pentavalentes. Tratamento.

\begin{abstract}
Efficacy and safety of meglumine antimoniate and sodium stibogluconate BP88R were compared in cutaneous leishmaniasis treatment in Corte de Pedra, Bahia, an endemic area of leishmaniasis due to Leishmania (Viannia) braziliensis. An open trial was developed with one hundred twenty seven patients who were diagnosed based on clinical criteria and Montenegro's skin test. Fifty eight patients were treated with meglumine antimoniate and 69 received sodium stibogluconate. Both groups received $20 \mathrm{mg} / \mathrm{Sbv} / \mathrm{kg} /$ day for 20 days. Patients were followed every ten days during treatment and every month thereafter for three months. Sixty two percent patients cured with meglumine antimoniate and 55\% cured with sodium stibogluconate $(p=0,42)$. Headache was more frequent during the first half of treatment in patients receiving sodium stibogluconate $(p=0,026)$. During the second half, patients treated with sodium stibogluconate showed a greater frequency of myalgia/arthralgia $(p=0,004)$ and abdominal pain/anorexia $(p=0,004)$. Three patients treated with sodium stibogluconate had severe side effects.
\end{abstract}

Key-words: Cutaneous leishmaniasis. Meglumine antimoniate. Sodium stibogluconate. Pentavalent antimonials. Treatment.

Nucleo de Medicina Tropical e Nutrição da Universidade de Brasilia, Brasilia, DF, Brasil.

Endereço para correspondência: Drª Ana Cristina Rodrigues Saldanha. Núcleo de Medicina Tropical, Universidade de Brasília, Campus Universitário Asa Norte, 70919-970 Brasília, DF, Brasil. Fax: 5561 273-2811.

Recebido para publicação 10/6/98. 
A complexidade da interação entre os elos da clássica tríade epidemiológica: parasitahospedeiro-vetor, no contexto do meio-ambiente, tem restringido as estratégias dos programas de controle à detecção e tratamento precoce dos casos humanos 10. Apesar das limitações atribuídas ao seu uso, os antimoniais pentavalentes permanecem como drogas de primeira escolha no tratamento da leishmaniose tegumentar americana (LTA). Atualmente duas formulações destes compostos encontram-se disponíveis: o antimoniato de meglumina (Rhodia, São Paulo, SP. Brasil) na rede pública brasileira e estibogluconato de sódio (PentostamR, Wellcome Foundation, Londres), utilizado em larga escala nos países de língua inglesa. Com o objetivo de reduzir os custos do tratamento das leishmanioses, outros países como a Índia e a China passaram a produzir estibogluconato de sódio. Entretanto, relatos sobre a experiência com a utilização destes produtos não se encontram na literatura mundial. Em 1996 o Ministério da Saúde do Brasil adquiriu um lote de estibogluconato de sódio de fabricação chinesa (Estibogluconato de sódio BP88R Shandong Xinhua, China) e a inexistência de estudos comparativos entre este e o antimoniato de meglumina, motivaram a realização do presente trabalho.

\section{PACIENTES E MÉTODOS}

Acompanharam-se duas coortes de pacientes com leishmaniose cutânea localizada (LCL), no distrito de Corte de Pedra, Presidente Tancredo Neves, Bahia, Brasil, área de reconhecida endemicidade de LTA, causada pela Leishmania (Viannia) braziliensis7. Para o cálculo da amostra assumiu-se que não existiria diferença entre a eficácia dos dois tratamentos propostos, estabelecendo-se um número ideal de 70 pacientes por grupo, incluindo $10 \%$ de perdas 5 . Este número permitiu detectar diferenças na incidência de efeitos colaterais maiores de $15 \%$ ( $\alpha=0,05$ e poder de $80 \%$ ). Foram incluídos 138 dos 279 casos de LTA atendidos no Centro de Saúde de Corte de Pedra, no período determinado para o estudo, que preencheram os seguintes critérios: idade entre 7 e 60 anos, quadro clínico compatível com LCL (máximo de quatro lesões), tempo de doença entre um e seis meses, ausência de comprometimento mucoso e de história de leishmaniose. Além dos critérios clínicos o paciente deveria apresentar Intradermorreação de Montenegro (IDRM) positiva e/ou pelo menos um dos seguintes exames diagnósticos positivos: visualização de amastigotas no exame direto por aposição de fragmento de biópsia de lesão cutânea ou aspecto histopatológico compatível com LTA. A IDRM foi realizada segundo a técnica preconizada pela Organização Mundial da Saúde utilizando-se como antígeno Leishmania (Leishmania) amazonensis-MHOM/BR/86/BA 125, 250ug/ml10. Foram excluídos pacientes tratados previamente com antimoniais pentavalentes ou drogas leishmanicidas nos últimos seis meses, aqueles com evidência clínica de alterações cardíacas, tuberculose, hanseníase, câncer, diabetes mellitus, hipertensão arterial sistêmica não controlada, comprometimento vascular periférico, história de alcoolismo, tratamento com corticosteróides ou outro imunossupressor e grávidas. Os pacientes que apresentaram efeitos colaterais graves que impediram a continuidade do tratamento foram incluídos na análise como falhas da terapêutica. Foram retirados do estudo os pacientes com falta de adesão ao protocolo (falha na administração de quatro doses consecutivas ou necessidade de um período superior a 24 dias para realização do tratamento completo de 20 doses) ou solicitação expressa do paciente para se retirar do estudo. Após o cadastro e realização dos procedimentos diagnósticos, os pacientes foram alocados sistematicamente em dois grupos submetidos aos seguintes esquemas terapêuticos:

Grupo I: Antimoniato de meglumina, (Rhodia, São Paulo, SP, Brasil), lote no 059L06, em ampolas de $5 \mathrm{ml}$, contendo $425 \mathrm{mg}$ de SbV.

Grupo II: Estibogluconato de sódio BP 88R (Shandong Xinhua, China) lote de no 96208, em ampolas de $5 \mathrm{ml}$, contendo $500 \mathrm{mg}$ de $\mathrm{SbV}$.

Em ambos os grupos utilizou-se a dose de $20 \mathrm{mg} / \mathrm{Sb} V / \mathrm{kg} / \mathrm{dia}$, aplicação única diária, durante 20 dias, via intravenosa ou intramuscular. A via intramuscular foi utilizada, como via alternativa, nunca exclusiva, quando o paciente não teve facilidades para a aplicação intravenosa. Ambas as drogas foram acondicionadas em geladeira a $4^{\circ} \mathrm{C}$. As avaliações foram realizadas nos dias 1 , $10,20,50,80$ e 110 a partir do primeiro dia de tratamento. $\mathrm{O}$ objetivo principal foi registrar a presença de edema, eritema, epitelização parcial ou completa das lesões. Estes parâmetros foram utilizados na determinação de cura clínica, definida como a ausência completa de qualquer 
sinal de inflamação na presença de cicatriz completamente epitelizada.

Quatro ampolas de cada um dos antimoniais pentavalentes utilizados no estudo foram analisadas para determinação de osmolaridade, $\mathrm{pH}$ e dosagem de antimônio total e trivalente11.

Os dados foram analisados utilizando o programa Epi-Info 6.04. Para todas as comparações foi estabelecido um limite de significância igual a 0,05. Para comparar proporções utilizou-se o teste do Chi-quadrado e para comparar médias T de student.

O estudo cumpriu com os requisitos exigidos pela Resolução do Conselho Nacional de Saúde 196/96 e foi aprovado pelo Comitê de ética em pesquisa envolvendo seres humanos da Faculdade de Ciências da Saúde da Universidade de Brasília.

\section{RESULTADOS}

A análise da homogeneidade entre os dois grupos terapêuticos mostrou que eram comparáveis, quando consideradas potenciais variáveis de confusão (Tabela 1). A amostra utilizada para estabelecer a eficácia dos dois medicamentos foi constituída por 127 pacientes, 58 no grupo I e 69 no grupo II. Observou-se uma incidência acumulada de cura clínica em 36 pacientes $(62 \%)$ do grupo I e em $38(55 \%)$ do grupo II $(p=0,42 ; R R=1,13 ; \mathrm{IC} 95 \%=0,84<$

Tabela 1 - Análise da homogeneidade entre os grupos submetidos à terapêutica com antimoniato de meglumina (Grupo I) e estibogluconato de sódio (Grupoll) em relação a aspectos clínicos e variações no tratamento

\begin{tabular}{|c|c|c|c|}
\hline variáveis & $\begin{array}{c}\text { Grupo I } \\
\mathrm{n}=58 \text { (DP) }\end{array}$ & $\begin{array}{c}\text { Grupo II } \\
\mathrm{n}=69 \text { (DP) }\end{array}$ & $P$ \\
\hline \multicolumn{4}{|l|}{ Clínicas } \\
\hline sexo $M / F$ & $39 / 19$ & $41 / 28$ & $0,36^{*}$ \\
\hline idade-média-anos & $23,8(11,7)$ & $24,6(11,4)$ & $0,73^{* *}$ \\
\hline \multicolumn{4}{|l|}{ número de lesões } \\
\hline únicas/múltiplas & $40 / 18$ & $52 / 17$ & $0,42^{*}$ \\
\hline \multicolumn{4}{|l|}{ Localização*** } \\
\hline membros inferiores & 49 & 58 & $0,64^{*}$ \\
\hline outro local & 30 & 41 & \\
\hline Área da lesão-média-cm² & $3,6(4,1)$ & $3,3(3,4)$ & $0,75^{\star *}$ \\
\hline Tempo de doença-média-semanas & $5,6(4,9)$ & $5,7(3,7)$ & $0,85^{* *}$ \\
\hline \multicolumn{4}{|l|}{ Terapêuticas } \\
\hline Percentagem de doses não contínuas-média & $5(6,1)$ & $6,1(9,8)$ & $0,47^{* *}$ \\
\hline
\end{tabular}

$R R<1,51)$. A incidência acumulada de cura clínica após o primeiro mês do final do tratamento foi igual a $12 \%$ e $7,2 \%$ nos pacientes dos grupos I e II, respectivamente $(p=0,35$; $R R=1,67 ;$ IC95\% $=0,56<R R<4,97)$. No segundo mês de seguimento observou-se um índice acumulado de cura clínica de 38\% para o grupo I e de $21 \%$ para o grupo II ( $p=0,02$; $R R=1,87 ;$ IC95\% = 1,06 < RR < 3,31). A análise da ocorrência de efeitos colaterais, independente do dia de avaliação, demonstrou uma incidência acumulada de $70,7 \%$ no grupo I e de $76,8 \%$ no grupo II ( $p=0,43$; RR =0,92, IC95\%=0,75< $R R<1,14)$. Três pacientes submetidos a tratamento com o estibogluconato de sódio apresentaram efeitos colaterais graves: exantema máculo-papular extenso; artralgia grave e extrasístoles bigeminadas, polimorfas e polifocais, tornando necessária a interrupção do esquema terapêutico. Seis pacientes do grupo II relataram parestesias e nenhum do grupo I. Avaliando-se eventos com possível correlação clínica, detectouse diferença estatisticamente significativa na incidência de manifestações sindrômicas músculo-esqueléticas (artralgia e mialgia) e em manifestações relacionadas ao aparelho digestivo (anorexia e dor abdominal), ocorrendo com maior freqüência nos pacientes do grupo II. A análise da diferença de incidência dos efeitos colaterais entre os dois grupos está demonstrada nas Tabelas 2 e 3 . 
Tabela 2 - Efeitos colaterais nos dois grupos terapêuticos no décimo dia de tratamento.

\begin{tabular}{|c|c|c|c|c|c|c|c|}
\hline \multirow[b]{2}{*}{ Efeito colateral } & \multicolumn{2}{|c|}{ Grupo I $(n=58)$} & \multicolumn{2}{|c|}{ Grupo II $(n=69)$} & \multirow[b]{2}{*}{$\mathrm{P}$} & \multirow[b]{2}{*}{$\mathrm{RR}^{\star \star \star}$} & \multirow[b]{2}{*}{ IC $95 \%$} \\
\hline & $\mathrm{n}^{\circ}$ & $\%$ & $\mathrm{n}^{\circ}$ & $\%$ & & & \\
\hline Mialgia/ artralgia & 7 & 12,0 & 17 & 24,6 & $0,072^{*}$ & 0,49 & $0,22<R R<1,10$ \\
\hline Cefaléia & 8 & 13,7 & 21 & 30,4 & 0,026 * & 0,45 & $0,22<R R<0,95$ \\
\hline Palpitação & 2 & 3,4 & 4 & 5,7 & 0,42 ** & 0,59 & $0,11<\mathrm{RR}<3,13$ \\
\hline Febre & 13 & 22,4 & 17 & 24,6 & 0,76 * & 0,91 & $0,48<\mathrm{RR}<1,71$ \\
\hline Dor abdominal/anorexia & 10 & 1,2 & 20 & 29,0 & 0,12 * & 0,59 & $0,30<R R<1,17$ \\
\hline
\end{tabular}

${ }^{*}$ Chi- quadrado; ${ }^{* *}$ Teste exato de Fisher; ${ }^{* * *}$ Risco relativo (exposição: antimoniato de meglumina).

Tabela 3 - Efeitos colaterais nos dois grupos terapêuticos no vigésimo dia de tratamento.

\begin{tabular}{|c|c|c|c|c|c|c|c|}
\hline \multirow[b]{2}{*}{ Efeito colateral } & \multicolumn{2}{|c|}{ Grupo I $(n=58)$} & \multicolumn{2}{|c|}{ Grupo II $(n=69)$} & \multirow[b]{2}{*}{$\mathrm{P}$} & \multirow[b]{2}{*}{$\mathrm{RR}^{\star \star *}$} & \multirow[b]{2}{*}{ IC 95\% } \\
\hline & $\mathrm{n}^{0}$ & $\%$ & $\mathrm{n}^{0}$ & $\%$ & & & \\
\hline Mialgia/artralgia & 27 & 46,5 & 48 & 71,6 & 0,004 & 0,65 & $0,47<R R<0,89$ \\
\hline Cefaléia & 9 & 15,5 & 20 & 29,8 & 0,059 & 0,52 & $0,26<R R<1,05$ \\
\hline Palpitação & 4 & 6,8 & 5 & 7,4 & 0,90 & 0,92 & $0,26<\mathrm{RR}<3,28$ \\
\hline Febre & 5 & 8,0 & 9 & 13,4 & 0,39 & 0,64 & $0,23<\mathrm{RR}<1,81$ \\
\hline Dor abdominal/anorexia & 18 & 31,0 & 38 & 56,7 & 0,004 & 0,55 & $0,35<\mathrm{RR}<0,85$ \\
\hline Outros ${ }^{* * *}$ & 2 & 3,0 & 14 & 20,8 & 0,003 & 0,17 & $0,04<\mathrm{RR}<0,70$ \\
\hline
\end{tabular}

*.Chi-quadrado. ${ }^{* *}$ Risco Relativo (exposição: antimoniato de meglumina). "**Náuseas, vômitos, "gosto amargo", hiperemia e ardor conjuntival.

Em relação à dosagem de SbV e SbllI, os valores observados foram compatíveis com as especificações de cada fabricante. A média de Sblll e Sb total por ampola de antimoniato de meglumina, foi $0,30 \mathrm{mg} / \mathrm{ml}$ e $80,24 \mathrm{mg} / \mathrm{ml}$, respectivamente. A média de Sblll e Sb total por ampola de estibogluconato de sódio foi $0,01 \mathrm{mg} / \mathrm{ml}$ e $106,7 \mathrm{mg} / \mathrm{ml}$, respectivamente. O valor médio do $\mathrm{pH}$ do antimoniato de meglumina foi 6,5 e 5,8 para o estibogluconato de sódio. O valor médio da osmolaridade do antimoniato de meglumina foi 783,2 mosM (716-866mosM) e a do estibogluconato de sódio foi 1049,2mosM (915 a $1274 \mathrm{mosM}$ ).

\section{DISCUSSÃO}

A diferença na metodologia entre os diversos ensaios clínicos realizados com os antimoniais pentavalentes dificulta a comparação da eficácia obtida em cada um deles. É conhecida a diferença de susceptibilidade entre as diversas espécies e cepas de Leishmania, o que torna ainda mais difícil a comparação dos resultados obtidos em regiões geográficas distintas. Alguns autores têm realizado estudos comparativos limitados entre antimoniato de meglumina (GlucantimeR) e estibogluconato de sódio (PentostamR) nas diferentes formas clínicas da LTA, observando resultados semelhantes com as duas drogas 289.

A eficácia comparável observada entre as drogas utilizadas pode ser explicada pelo fato de serem, ambas, pertencentes a uma mesma classe farmacológica, apresentando mecanismos de ação e comportamento farmacocinético teoricamente semelhantes 134 . A diferença observada dois meses após o tratamento, permite afirmar que nos pacientes tratados com antimoniato de meglumina a resolução das lesões foi mais rápida. O mecanismo pelo qual o antimoniato de meglumina produziria cura clínica em um período de tempo mais reduzido, não têm sido descrito na literatura.

A análise quantitativa global dos efeitos colaterais não mostrou diferenças com significância estatística entre as duas drogas, porém, somente no grupo tratado com o estibogluconato de sódio houve necessidade de interrupção do tratamento devido a efeitos colaterais graves. Em dois destes três pacientes, as alterações ocorreram na primeira metade do tratamento, o que demonstra que além do efeito cumulativo da droga, outros mecanismos devem estar relacionados à sua toxicidade. Manifestações músculo-esqueléticas, anorexia 
associada a dor abdominal, e cefaléia foram mais freqüentes na segunda metade do tratamento, ocorrendo principalmente nos pacientes tratados com estibogluconato de sódio. A análise de amostras dos dois compostos utilizados no presente trabalho nos permite afirmar com relativa segurança que não houve diferença na quantidade de antimônio administrada nos dois grupos terapêuticos excluindo a possibilidade de que alterações nesta variável explicassem as diferenças observadas na frequência de efeitos colaterais como foi sugerido na literatura 46 . A ocorrência de efeitos associada às doses convencionais, como foi observado neste estudo, ressalta a necessidade de investigar sobre os mecanismos que contribuem para o aparecimento destas alterações. A análise dos nossos resultados permite afirmar que apesar da igualdade de eficácia entre $o$ antimoniato de meglumina e o estibogluconato de sódio, a freqüência e a gravidade dos efeitos colaterais com o uso do estibogluconato de sódio de fabricação chinesa, são maiores. Desta forma o estibogluconato de sódio não mostrou ser uma alternativa para o tratamento da LTA.

\section{AGRADECIMENTOS}

Ao Professor Reynaldo Dietze que facilitou a análise dos antimoniais na Universidade Federal do Espírito Santo, à Fundação Nacional de Saúde, Ministério da Saúde por ceder os lotes dos medicamentos utilizados e ao Sr. Ednaldo Lima do Lago da FNS pela ajuda no desenvolvimento do trabalho de campo.

\section{REFERÊNCIAS BIBLIOGRÁFICAS}

1. Berman JD. Chemotherapy for Leishmaniasis: Biochemical mechanisms, clinical efficacy and future strategies. Reviews of Infectious Diseases 10:560-586, 1988.

2. Costa JML. Estudo clínico epidemiológico de um surto epidêmico de leishmaniose tegumentar americana em Corte de Pedra-BA. Tese de Mestrado, Universidade de Brasília, Brasília, DF, 1986.

3. Chulay JD, Fleckenstein L, Smith DH. Pharmacokinetics of antimony during treatment of visceral leismaniasis with sodium stibogluconate or meglumine antimoniate. Transactions of the Royal Society of Tropical Medicine and Hygiene 82:69-72, 1988.

4. Marsden PD. Pentavalent antimonials: old drugs for new diseases. Revista da Sociedade Brasileira de Medicina Tropical 18:187-198, 1985.

5. Pocock SJ. Clinical trials. Jonh Wiley \& Sons, Chichester, Great Britain, 1987.

6. Romero GAS, Oliveira MRF, Correia D, Marsden PD. Características físico-químicas do antimoniato de meglumina em diferentes condições de armazenamento. Revista da Sociedade Brasileira de Medicina Tropical 29:461-465, 1996.
7. Rosa AC, Cuba CC, Vexanat A, Barreto AC, Marsden PD. Predominance of Leishmania braziliensis braziliensis in the regions of Três Braços and Corte de Pedra, BahiaBrasil. Transactions of Royal Society of Tropical Medicine and Hygiene 82:409-410, 1988.

8. Sáenz RE, Narváez E. Evaluation of the effectivenes and toxicity of pentostam and glucantime in the treatment of cutaneous leishmaniasis. Revista Médica de Panamá 12:148-157, 1987.

9. Sampaio RNR. Tratamento hospitalar da leishmaniose cutâneo-mucosa. Tese de Mestrado. Universidade Federal de Minas Gerais, Belo Horizonte, MG, 1984.

10. World Health Organization. Control of Leishmaniasis. Report of a WHO Expert Committe. Technical Report Series. 793, 1990.

11. Yonehara N, Fugi T, Sakamoto H, Kamada M. Differential determination of antimony (III) and antimony (V) by indirect espectrophotometry with uromium (VI) and diphenylcarbazide after reduction of antimony (V). Analytica Chimica Acta 199:129-135, 1987. 